\title{
Phosphatase of regenerating liver-3 as a prognostic biomarker in histologically node-negative gastric cancer
}

\author{
AKIRA OOKI, KEISHI YAMASHITA, SHIRO KIKUCHI, SHINICHI SAKURAMOTO, \\ NATSUYA KATADA and MASAHIKO WATANABE \\ Department of Surgery, Kitasato University Hospital, Kitasato 1-15-1, Sagamihara 228-8555, Kanagawa, Japan
}

Received January 29, 2009; Accepted March 12, 2009

DOI: $10.3892 /$ or_00000376

\begin{abstract}
Phosphatase of regenerating liver-3 (PRL-3) has received attention as a molecule associated with metastasis in various tumor types, including gastric cancer. However, its clinical utility as a biomarker remains unclear in primary gastric cancer. The present study included 173 patients with primary gastric cancer who underwent gastrectomy with regional lymphadenectomy at the Kitasato University Hospital. All patients were informative for the well-characterized clinicopathological variables, including patient outcome. We assessed the potential as a clinically applicable biomarker using immunohistochemistry. PRL-3 overexpression was detected in $78(45 \%)$ of 173 primary tumor tissues and was an independent predictor of lymph node metastasis on multivariate logistic regression analysis (odds ratio $=9.32$; $\mathrm{P}<0.0001)$. PRL-3 overexpression in primary tumor had significant prognostic implication $(\mathrm{P}=0.0009)$ and was also an independent prognostic factor (hazard ratio=4.39; $\mathrm{P}=0.006)$ in the histologically node-negative patients after curative resection, but not in the histologically node-positive patients. Moreover, in advanced gastric cancer with stage I disease, PRL-3 overexpression inversely affected patient outcome $(\mathrm{P}=0.02)$ and showed a characteristic of stage II disease from a prognostic point of view. We demonstrated for the first time that PRL-3 expression in primary tumor could predict the outcome of patients with histologically node-negative gastric cancer. We propose that PRL-3 expression can have a clinical potential as a prognostic biomarker that may facilitate the development of adjuvant chemotherapy for advanced gastric cancer with stage I disease.
\end{abstract}

\section{Introduction}

Gastric cancer is the fourth most common cancer and the second leading cause of cancer-related death worldwide (1).

Correspondence to: Professor Masahiko Watanabe, Department of Surgery, Medical School, Kitasato University, Kitasato 1-15-1, Sagamihara 228-8555, Kanagawa, Japan

E-mail: gekaw@med.kitasato-u.ac.jp

Key words: phosphatase of regenerating liver-3, lymph node metastasis, prognosis
Geographic areas with a high risk of gastric cancer include East Asian countries, where the age-standardized incidence rate is $>20$ per $1,000,000$ (2). Recent improvements in diagnostic tools and methods have facilitated detection of early gastric cancer and thereby excellent long-term survival. However, the outcome of patients with advanced disease at the time of diagnosis remains poor. Metastasis is a multistep process, involving basement membrane destruction, local invasion, intravasation and survival in the bloodstream, extravasation into distant organs, and survival plus proliferation at the metastatic site (3), and is a primary cause of mortality. Therefore, identifying prominent factors for metastasis would offer further understanding and treatment strategies in gastric cancer.

The protein tyrosine phosphatases (PTPs) form a large family of enzymes that serve as key regulatory components in signal transduction pathways (4). The phosphatases of regenerating liver (PRL-1, -2 , and -3 ), belonging to a small class of PTP superfamily, have a unique $\mathrm{COOH}$-terminal prenylation motif, which critically affects their cellular localization and function (5). All PRL proteins share significant amino acid sequence homology, but PRL-3, unlike PRL-1 and -2 with ubiquitous expression, is expressed predominantly in muscle and heart among normal human adult tissues (6-8).

Among human tumor tissues, PRL-3 was first identified to be specifically overexpressed in liver metastases derived from colorectal cancer using serial analysis of gene expression (SAGE) technology (9), and subsequently an accumulating number of studies demonstrated PRL-3 overexpression in various tumor types (10-14). Enforced PRL-3 expression can promote invasion, migration, and angiogenesis in cell culture and mouse model systems (15-19), whereas knockdown of endogenous PRL-3 by small interfering RNA (siRNA) or transfection with catalytically inactive mutants can abrogate these properties $(11,20,21)$. Therefore, PRL-3 has deserved attention as a molecule associated with metastasis in various tumor types.

In primary gastric cancer, PRL-3 overexpression was observed in 36.2-70.4\% using immunohistochemistry or in situ hybridization and was associated with lymph node metastasis or peritoneal metastasis $(10,22-24)$, implying that PRL-3 may serve as a biomarker for broadening treatment strategies in clinical practice, but has not been previously assessed. Therefore, in the present study, we examined the 
characteristics of PRL-3 expression in primary gastric cancer and assessed its potential as a clinically applicable biomarker.

\section{Materials and methods}

Tissue samples. Formalin-fixed, paraffin-embedded tissue samples were obtained from 173 randomly selected patients with primary gastric cancer who underwent gastrectomy with regional lymphadenectomy at the Kitasato University Hospital from 1999 to 2003. Surgery was done according to the gastric cancer treatment guidelines in Japan (25). All the histopathologic examinations were done by histopathologists according to the 13th edition of the Japanese Classification of Gastric Carcinoma (JCGC) (26).

JCGC staging system is designed by using depth of invasion ( $\mathrm{T}$-factor), lymph node metastasis ( $\mathrm{N}$-factor), liver metastasis (H-factor), peritoneal metastasis ( $\mathrm{P}$-factor), other distant metastasis (M-factor), and peritoneal cytology (CY-factor), and the presence of any of $\mathrm{H}$-factor, P-factor, $\mathrm{M}$-factor, or CY-factor is classified in stage IV disease. Stage I disease has three subgroups: T1N0, T1N1, and T2N0. $\mathrm{T}$-factor in JCGC is identical to that in the International Union Against Cancer (UICC) (27).

Early gastric cancer is defined as cancer confined to the mucosa or submucosa (T1), irrespective of presence or absence of lymph node metastases. Advanced gastric cancer is defined as cancer invading the muscularis propria or deeper (T2 to T4). Namely, T2N0 represents advanced gastric cancer with stage I disease. We excluded patients who had any of the following: insufficient regional lymphadenectomy, neoadjuvant therapy, other primary malignancy, death of other causes, or insufficient information for the clinicopathological variables, including outcome. The mean age of the patients was 60 years (range: $31-81$ years). Of the 173 patients, the disease stage according to the JCGC was I in 76 patients, II in 32 , III in 40 , and IV in 25 , respectively. All patients with stage I-III disease underwent curative resection.

Patients with stage IV disease were not identified for any distant metastasis by preoperative imaging techniques, such as dynamic computed tomography and ultrasound, and by perioperative palpation, and thereby underwent gastrectomy with regional lymphadenectomy. Among stage IV disease, 5 patients had positive CY by cytological examination of peritoneal washes at perioperation regardless of negative lymph node metastasis, which was no longer a curative resection. On prognostic analysis in node-negative patients after curative resection, we therefore excluded these 5 patients. Detailed information on the 173 patients is summarized in Table I. The present study was approved by the Ethics Committee of Kitasato University.

Immunohistochemistry. Sections (4 $\mu \mathrm{m})$ were deparaffinized in xylene and rehydrated through graded ethanol. After antigen retrieval was performed with proteinase $\mathrm{K}$ (Dakocytomation, Inc., Carpinteria, CA) for $3 \mathrm{~min}$, endogenous peroxidase activity was quenched with methanol containing $3 \%$ hydrogen peroxide for $5 \mathrm{~min}$. The sections were blocked with $1 \%$ bovine serum albumin for $30 \mathrm{~min}$, followed by incubation with the primary mouse monoclonal PRL-3 antibody
(MAB3219, R\&D Systems, Inc., MN) overnight at $4^{\circ} \mathrm{C}$. We previously showed that this antibody was most sensitive for detection of PRL-3 expression (28). After washing in TBS-T consisting of $25 \mathrm{mmol} / \mathrm{l}$ Tris- $\mathrm{HCl} \mathrm{PH} \mathrm{7.4,} 150 \mathrm{mmol} / \mathrm{l} \mathrm{NaCl}$, and $0.1 \%$ Tween-20 three times, the sections were incubated with goat anti-mouse IgG secondary antibody combined with horseradish peroxidase (Nichirei Biosciences, Inc., Tokyo, Japan) for $60 \mathrm{~min}$. Chromogenic fixation was performed in a solution of 3,3-diamino-benzidine tetrahydrochloride at room temperature for $3 \mathrm{~min}$, and the sections were counterstained with Mayer's hematoxylin. For the immunohistochemical staining procedure, slides without the primary antibody as a negative control and with liver metastases derived from colorectal cancer as a positive control were used.

Immunohistochemical staining was evaluated by two independent investigators without any knowledge of the clinical information for the corresponding cases. The scoring of immunoreactivity was conducted as follows: score 0 , almost no immunoreactive cancer cells; score 1, faint intensity or $<10 \%$ staining cells; score 2, moderate intensity; score 3, intensity as strong as positive control. Score 2 and 3 were defined as PRL-3 overexpression.

Statistical analysis. The Chi-square test or Mann-Whitney $\mathrm{U}$ test was used for the correlation between PRL-3 expression and the clinicopathological variables. The Kaplan-Meier method was used to estimate the cumulative survival rates, and the difference in survival rates was assessed with the use of the log-rank test (29). All deaths of patients were cancerrelated, and the 5-year disease specific survival (DSS) was measured from the date of surgery to the date of death or the last follow-up. The data for patients who survived for $>60$ months were censored at the time of 60 months. The variables suggested to be prognostic factors on univariate analysis $(\mathrm{P}<0.05)$ were subjected to multivariate analysis using the Cox proportional-hazard regression model (30). A logistic regression model was used to identify the most prominent factors for lymph node metastasis. $\mathrm{P}<0.05$ was considered to indicate statistical significance. All the statistical analyses were conducted with the SAS software package StatView version 5.0 (SAS Institute, Cary, NC, USA).

\section{Results}

Expression frequencies of PRL-3 and correlation with clinicopathological variables. Immunohistochemical analysis was performed to evaluate the frequency of PRL-3 protein overexpression in 173 primary gastric tumor tissues. Representative examples of immunohistochemical staining for PRL-3 are shown in Fig. 1. PRL-3 overexpression was detected in 78 $(45 \%)$ of 173 primary tumor tissues, but not in histological adjacent normal tissues (data not shown). We also examined 40 matched pairs of primary tumor and corresponding metastatic lymph node tissues selected randomly from 83 node-positive patients. PRL-3 overexpression was found in $29(72.5 \%)$ of 40 primary tissues and $37(92.5 \%)$ of the corresponding metastatic lymph node tissues. This difference was statistically significant $(\mathrm{P}=0.04)$. Next, we analyzed the correlation between PRL-3 overexpression and clinicopathological variables (Table I). PRL-3 overexpression was 
Table I. Correlation between PRL-3 and clinicopathologic variables in 173 patients with gastric cancer.

\begin{tabular}{|c|c|c|c|c|}
\hline \multirow[b]{2}{*}{ Variables } & \multirow[b]{2}{*}{ Total number } & \multicolumn{2}{|c|}{ RL-3 expression } & \multirow[b]{2}{*}{ P-value } \\
\hline & & $\begin{array}{c}\text { Negativity } \\
\text { number (\%) }\end{array}$ & $\begin{array}{c}\text { Positivity } \\
\text { number (\%) }\end{array}$ & \\
\hline Age (years) & & & & NS \\
\hline$<60$ & 80 & $42(53)$ & $38(47)$ & \\
\hline$\geq 60$ & 93 & $36(39)$ & $57(61)$ & \\
\hline Gender & & & & NS \\
\hline Male & 121 & $55(45)$ & $66(55)$ & \\
\hline Female & 52 & $23(44)$ & $29(56)$ & \\
\hline Main location & & & & NS \\
\hline Upper & 45 & $16(36)$ & $29(64)$ & \\
\hline Middle & 51 & $25(49)$ & $26(51)$ & \\
\hline Lower & 77 & $37(48)$ & $40(52)$ & \\
\hline Growth pattern ${ }^{\mathrm{a}}$ & & & & NS \\
\hline Expansive growth & 122 & $53(43)$ & $69(57)$ & \\
\hline Infiltrative growth & 51 & $25(49)$ & $26(51)$ & \\
\hline Lymphatic permeation & & & & 0.0007 \\
\hline Absence & 23 & $18(78)$ & $5(22)$ & \\
\hline Presence & 150 & $60(40)$ & $90(60)$ & \\
\hline Vascular permeation & & & & 0.01 \\
\hline Absence & 39 & $25(64)$ & $14(36)$ & \\
\hline Presence & 134 & $53(40)$ & $81(60)$ & \\
\hline Differentiation & & & & NS \\
\hline Well and moderate & 75 & $30(40)$ & $45(60)$ & \\
\hline Poor & 98 & $48(49)$ & $50(51)$ & \\
\hline Depth of invasion & & & & $\mathrm{NS}^{\mathrm{c}}$ \\
\hline $\mathrm{T} 1$ (m and sm) & 43 & $22(51)$ & $21(49)$ & \\
\hline $\mathrm{T} 2$ (mp and ss) & 79 & $39(49)$ & $40(51)$ & \\
\hline $\mathrm{T} 3(\mathrm{se})$ & 38 & $14(37)$ & $24(63)$ & \\
\hline T4 (si) & 13 & $3(23)$ & $10(77)$ & \\
\hline Lymph node metastasis & & & & $<0.0001$ \\
\hline Absence & 90 & $61(68)$ & $29(32)$ & \\
\hline Presence & 83 & $17(20)$ & $66(80)$ & \\
\hline $\mathrm{CY}^{\mathrm{b}}$ & & & & NS \\
\hline Absence & 98 & $45(46)$ & $53(54)$ & \\
\hline Presence & 18 & $6(33)$ & $12(67)$ & \\
\hline Stage according to the JCGC & & & & $<0.0001^{\circ}$ \\
\hline I (IA and IB) & 76 & $48(63)$ & $28(37)$ & \\
\hline II & 32 & $13(41)$ & $19(59)$ & \\
\hline III (IIIA and IIIB) & 40 & $9(23)$ & $31(77)$ & \\
\hline IV & 25 & $8(32)$ & $17(68)$ & \\
\hline
\end{tabular}

m, mucosa; sm, submucosa; mp, muscularis propria; ss, subserosa; se, serosa-exposed; si, serosa-infiltrating; CY, peritoneal cytology; JCGC, Japanese Classification of Gastric Carcinoma; NS, not significant. ${ }^{a}$ Expansive growth pattern is defined as the presence, as viewed under the microscope, of coherent tumor cell complexes with a clear margin; infiltrative growth pattern is defined as non-expansion. ${ }^{\mathrm{b}}$ Cytological examination using peritoneal washes obtained at the time of surgery was performed for patients diagnosed preoperatively as serosal invasion. ${ }^{\mathrm{c} M a n n-W h i t n e y ~} \mathrm{U}$ test, the remaining variables: Chi-square test.

significantly associated with JCGC stage $(\mathrm{P}<0.0001)$, lymph node metastasis $(\mathrm{P}<0.0001)$, lymphatic permeation $(\mathrm{P}=0.0007)$, and vascular permeation $(\mathrm{P}=0.01)$. These findings were almost consistent with previous studies, supporting the validity of PRL-3 overexpression defined in the present study $(10,22-24)$.
Univariate and multivariate logistic regression analyses were performed to identify the most prominent factors for lymph node metastasis because it is an important prognostic factor in gastric cancer (Table II). PRL-3 overexpression $(\mathrm{P}<0.0001)$, lymphatic permeation $(\mathrm{P}=0.0002)$, and growth pattern $(\mathrm{P}=0.03)$, suggested to have potential on the univariate 
HE
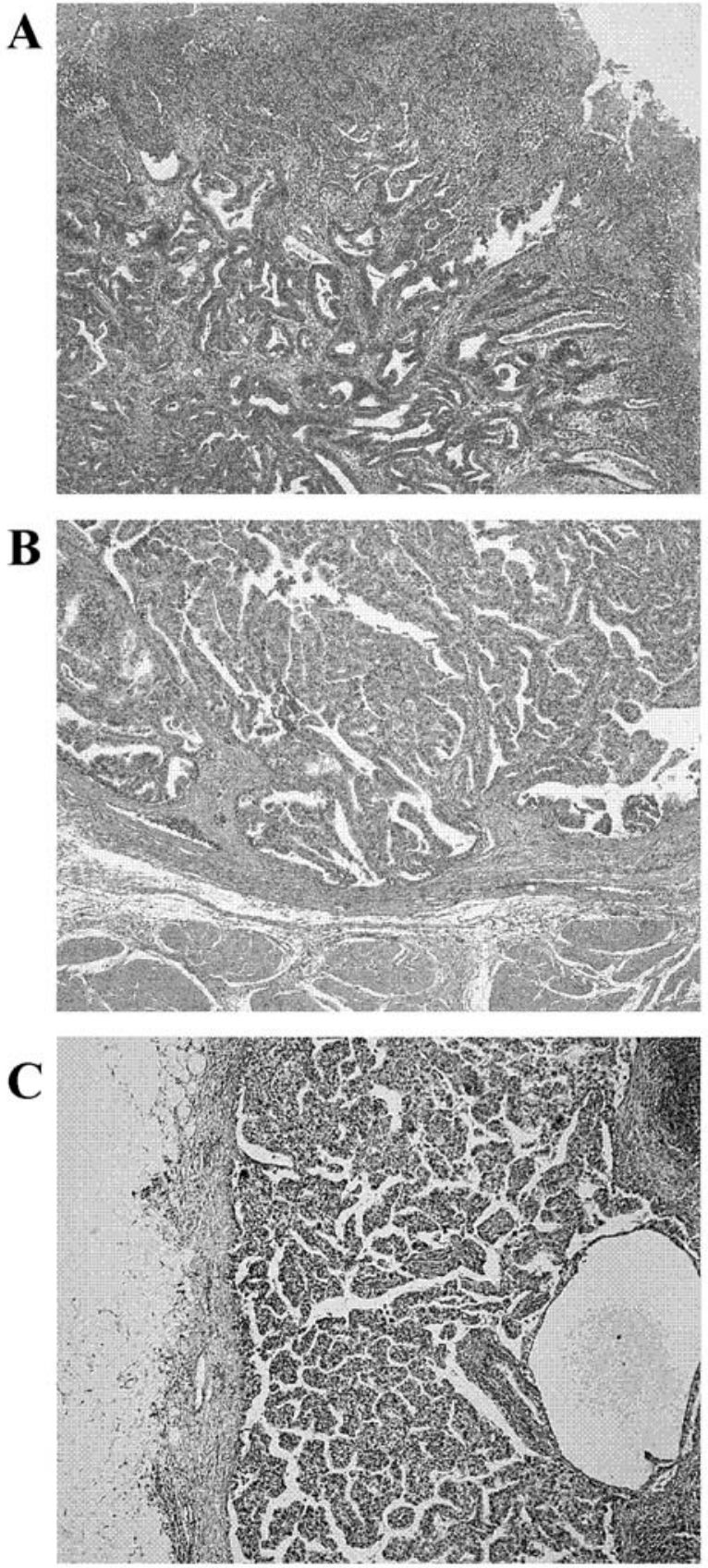

PRL-3
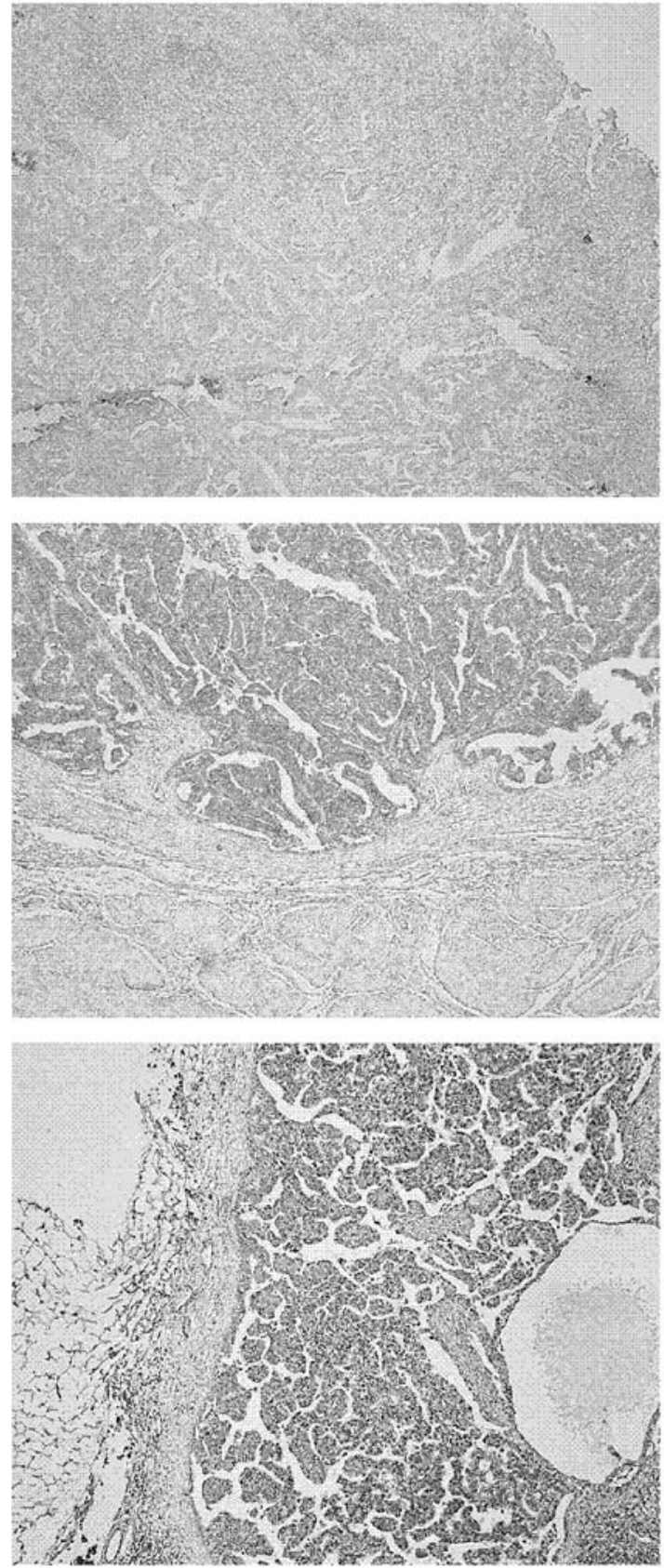

Figure 1. Representative expression of PRL-3 by immunohistochemical staining (right). (A) Negative expression of PRL-3 in primary tumor. Positive expression of PRL-3 in (B) primary tumor and (C) metastatic lymph node. Slides were counterstained with hematoxylin and eosin (left). Original magnification, $\mathrm{x} 40$.

analysis, were included in the multivariate analysis. Multivariate analysis showed that PRL-3 overexpression (odds ratio $[\mathrm{OR}]=9.32 ; \mathrm{P}<0.0001$ ) and lymphatic permeation $(\mathrm{OR}=3.23 ; \mathrm{P}=0.04)$ remained the only independent predictors of lymph node metastasis in gastric cancer.

PRL-3 as a prognostic biomarker in histologically nodenegative patients. To assess whether PRL-3 overexpression in primary tumor can serve as a prognostic biomarker, we performed both univariate and multivariate prognostic analyses (Table III). We did not include T-factor, N-factor, or CYfactor as variables in the multivariate prognostic analysis because the JCGC staging system is based on these factors. JCGC Stage $(\mathrm{P}<0.0001)$, PRL-3 overexpression $(\mathrm{P}<0.0001)$, vascular permeation $(\mathrm{P}<0.0001)$, lymphatic permeation $(\mathrm{P}=0.0005)$, and growth pattern $(\mathrm{P}=0.008)$, suggested to have prognostic potential on the univariate analysis, were included in the multivariate analysis. Multivariate analysis showed that JCGC stage $(\mathrm{P}<0.0001)$ and $\mathrm{PRL}-3$ overexpression (hazard ratio $[\mathrm{HR}]=2.74 ; \mathrm{P}<0.0001)$ remained the only independent predictors of prognosis in gastric cancer.

In previous studies, PRL-3 overexpression in primary tumor had an important prognostic implication in all patients with gastric cancer, but not in histologically node-positive 
Table II. Univariate and multivariate logistic regression analysis for lymph node metastasis in 173 patients with gastric cancer.

\begin{tabular}{|c|c|c|c|c|c|c|}
\hline \multirow[b]{2}{*}{ Variables } & \multicolumn{3}{|c|}{ Univariate analysis } & \multicolumn{3}{|c|}{ Multivariate analysis } \\
\hline & OR & $95 \% \mathrm{CI}$ & P-value & OR & $95 \% \mathrm{CI}$ & P-value \\
\hline PRL-3 expression (negativity/positivity) & 8.17 & $4.09-16.32$ & $<0.0001$ & 9.32 & $4.30-20.24$ & $<0.0001$ \\
\hline Lymphatic permeation (absence/presence) & 4.93 & $2.11-11.51$ & 0.0002 & 3.23 & $1.24-8.42$ & 0.04 \\
\hline Growth pattern (expansion/infiltration) & 2.09 & $1.07-4.07$ & 0.03 & 2.77 & $1.19-6.42$ & NS \\
\hline Vascular permeation (absence/presence) & 1.74 & $0.73-4.17$ & NS & - & - & - \\
\hline Differentiation (well or moderate/poor) & 1.46 & $0.80-2.67$ & NS & - & - & - \\
\hline Age $(<60$ years $/ \geq 60$ years $)$ & 1.25 & $0.69-2.27$ & NS & - & - & - \\
\hline Depth of invasion (T1, T2/T3, T4) & 1.13 & $0.80-1.59$ & NS & - & - & - \\
\hline Gender (male/female) & 0.80 & $0.42-1.53$ & NS & - & - & - \\
\hline
\end{tabular}

OR, odds ratio; CI, confidence interval; NS, not significant.

Table III. Univariate and multivariate prognostic analyses in 173 patients with gastric cancer.

\begin{tabular}{|c|c|c|c|c|c|}
\hline \multirow[b]{2}{*}{ Variables } & \multicolumn{2}{|c|}{ Univariate analysis } & \multicolumn{3}{|c|}{ Multivariate analysis } \\
\hline & DSS (months) & P-value & HR & $95 \% \mathrm{CI}$ & P-value ${ }^{b}$ \\
\hline Stage according to the JCGC & & $<0.0001$ & & & $<0.0001$ \\
\hline I (IA and IB) & 56 & \multicolumn{4}{|c|}{ Reference } \\
\hline II & 41 & & 2.44 & $1.04-5.72$ & 0.04 \\
\hline III (IIIA and IIIB) & 34 & & 3.32 & $1.52-7.24$ & 0.003 \\
\hline IV & 19 & & 10.07 & $4.54-22.34$ & $<0.0001$ \\
\hline \multicolumn{6}{|l|}{ PRL-3 expression } \\
\hline Negativity/positivity & $51 / 37$ & $<0.0001$ & 2.74 & $1.53-4.90$ & 0.0007 \\
\hline \multicolumn{6}{|l|}{ Lymphatic permeation } \\
\hline Absence/presence & $56 / 41$ & 0.0005 & 2.13 & $0.23-19.66$ & NS \\
\hline \multicolumn{6}{|l|}{ Vascular permeation } \\
\hline Absence/presence & $56 / 39$ & $<0.0001$ & 2.13 & $0.68-6.67$ & NS \\
\hline \multicolumn{6}{|l|}{ Growth pattern } \\
\hline Expansion/infiltration & $46 / 37$ & 0.008 & 1.25 & $0.74-2.10$ & NS \\
\hline \multicolumn{6}{|l|}{ Age (years) } \\
\hline$<60$ vs. $\geq 60$ & $45 / 41$ & NS & - & - & - \\
\hline \multicolumn{6}{|l|}{ Gender } \\
\hline Male/female & $42 / 45$ & NS & - & - & - \\
\hline Main location & & NS & - & - & - \\
\hline Upper & 37 & & & & \\
\hline Middle & 48 & & & & \\
\hline Lower & 43 & & & & \\
\hline \multicolumn{6}{|l|}{ Differentiation } \\
\hline Well or moderate/poor & $46 / 41$ & NS & - & - & - \\
\hline
\end{tabular}

DSS, disease-specific survival; HR, hazard ratio; CI, confidence interval; NS, not significant. ${ }^{\mathrm{a} L o g-r a n k}$ test; ${ }^{\mathrm{b}}$ Cox proportional hazard regression model.

patients $(23,24)$. While, the association between PRL-3 overexpression in primary tumor and outcome of histologically node-negative patients has not been reported to date. Hence, we investigated whether PRL-3 overexpression in primary tumor could predict patient outcome in the each subgroup with or without histological lymph node metastasis. PRL-3 overexpression did not have a significant prognostic implication in 83 histologically node-positive patients, as indicated in a previous study (24) (Fig. 2A). In contrast, PRL-3 overexpression was significantly associated with poor 
Table IV. Univariate and multivariate prognostic analyses in 85 node-negative patients after curative resection.

\begin{tabular}{|c|c|c|c|c|c|}
\hline \multirow[b]{2}{*}{ Variables } & \multicolumn{2}{|c|}{ Univariate analysis } & \multicolumn{3}{|c|}{ Multivariate analysis } \\
\hline & $\begin{array}{c}\text { Mean DSS } \\
\text { (months) }\end{array}$ & P-value ${ }^{a}$ & HR & $95 \% \mathrm{CI}$ & P-value ${ }^{b}$ \\
\hline \multicolumn{6}{|l|}{ PRL-3 expression } \\
\hline Negativity/positivity & $55 / 41$ & 0.0009 & 4.39 & $1.53-12.66$ & 0.006 \\
\hline Stage according to the JCGC & & 0.001 & & & NS \\
\hline I (IA and IB) & 56 & \multicolumn{4}{|c|}{ Reference } \\
\hline II & 39 & & 2.03 & $0.33-12.35$ & NS \\
\hline III (IIIA and IIIB) & 32 & & 3.45 & $0.85-13.96$ & NS \\
\hline \multicolumn{6}{|l|}{ Vascular permeation } \\
\hline Absence/presence & $57 / 48$ & 0.03 & 2.19 & $0.33-14.51$ & NS \\
\hline \multicolumn{6}{|l|}{ Lymphatic permeation } \\
\hline Absence/presence & $56 / 49$ & 0.04 & 1.91 & $0.14-26.33$ & NS \\
\hline \multicolumn{6}{|l|}{ Growth pattern } \\
\hline Expansion/infiltration & $53 / 46$ & 0.04 & 0.56 & $0.11-2.99$ & NS \\
\hline \multicolumn{6}{|l|}{ Age (years) } \\
\hline$<60$ vs. $\geq 60$ & $53 / 50$ & NS & - & - & - \\
\hline \multicolumn{6}{|l|}{ Gender } \\
\hline Male/female & $52 / 49$ & NS & - & - & - \\
\hline Main location & & NS & - & - & - \\
\hline Upper & 46 & & & & \\
\hline Middle & 54 & & & & \\
\hline Lower & 51 & & & & \\
\hline \multicolumn{6}{|l|}{ Differentiation } \\
\hline Well or moderate/poor & $53 / 50$ & NS & - & - & - \\
\hline
\end{tabular}

DSS, disease-specific survival; HR, hazard ratio; CI, confidence interval; NS, not significant. ${ }^{\mathrm{a} L o g-r a n k}$ test; ${ }^{\mathrm{b}}$ Cox proportional hazard regression model.

outcome in 85 histologically node-negative patients after curative resection $(\mathrm{P}=0.0009$, Fig. $2 \mathrm{~B})$. Moreover, PRL-3 overexpression was the only independent prognostic factor even in the node-negative patients after curative resection $(\mathrm{HR}=4.39 ; \mathrm{P}=0.006$, Table IV). These findings suggest that PRL-3 overexpression in primary tumor may have great potential as an aggressive biomarker in histologically nodenegative patients after curative resection.

Finally, we focused on prognostic potential of PRL-3 overexpression in each $\mathrm{T}$-factor among the node-negative patients after curative resection. In T1 disease (i.e., early gastric cancer, $n=26$ ), 5-year DSS rate was $100 \%$, implying that early gastric cancer has excellent outcome irrespective of the presence or absence of PRL-3 overexpression (data not shown). In T2 disease (i.e., advanced gastric cancer with stage I disease, $\mathrm{n}=37$ ), PRL-3 overexpression inversely affected the patient outcome $(\mathrm{P}=0.02)$ and showed a prognosis similar to that associated with stage II disease (Fig. 2C). In T3 to T4 disease $(n=22)$, PRL-3 overexpression was also significantly associated with poor outcome $(\mathrm{P}=0.04)$.

\section{Discussion}

Lymph node metastasis is considered as one of the most frequent and important prognostic factors in gastric cancer (31), and thus complete surgical resection with regional lymph node dissection has been a standard procedure for curative treatment. However, the Dutch Gastric Cancer Group reported that $\sim 20 \%$ of node-negative patients died due to disease recurrence within 3 years regardless of curative resection with D1 or D2 lymph node dissection (32). Therefore, prognostic markers are prerequisite to the management of treatment in node-negative patients after curative resection. Herein, we report for the first time that PRL-3 overexpression in primary tumor was able to predict the outcome of patients with histologically node-negative gastric cancer and serve as a clinically applicable biomarker.

In the present study, PRL-3 overexpression was an independent predictor of lymph node metastasis on multivariate logistic regression analysis in 173 patients studied (Table II). Surprisingly, in 85 histologically node-negative patients after 
A

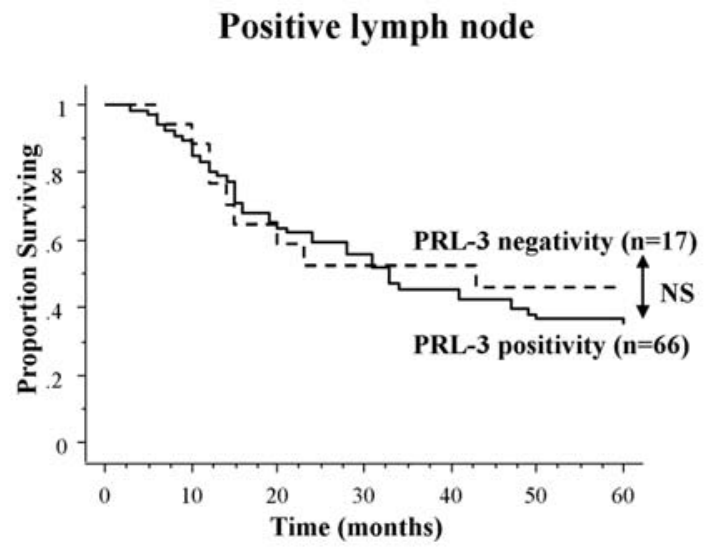

C

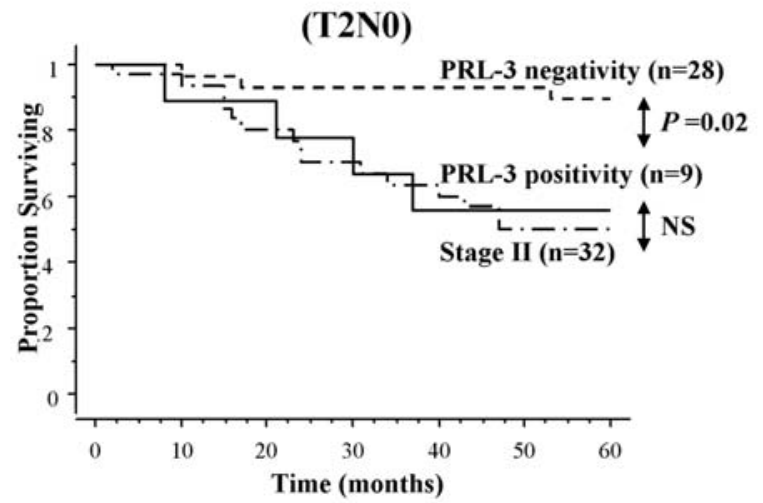

B

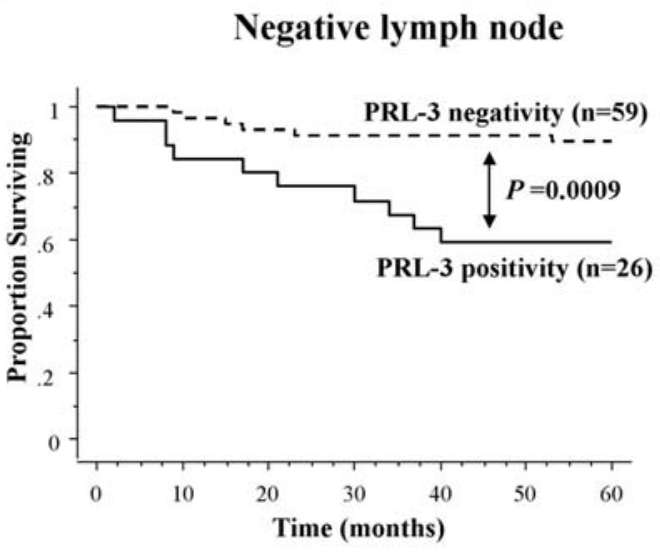

D

T3/T4N0

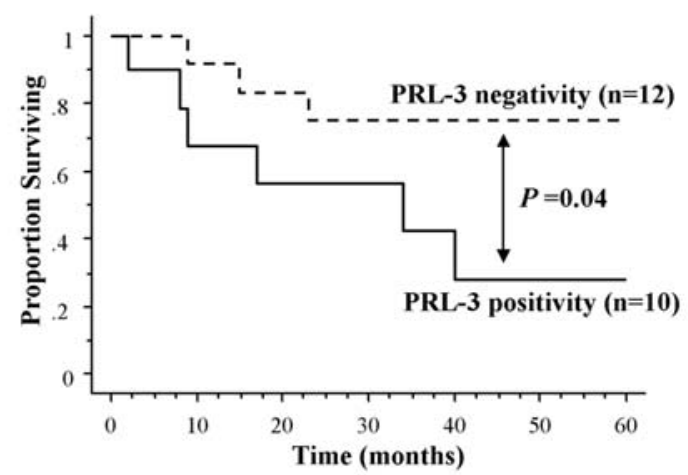

Figure 2. Prognostic analysis of PRL-3 expression in primary gastric tumor tissues. Kaplan-Meier curves for PRL-3 expression in primary tumor with (A) histologically positive lymph node (NS), (B) histologically-negative lymph node after curative resection (P=0.0009), (C) T2N0 (i.e., advanced gastric cancer with stage I disease, $\mathrm{P}=0.02)$, and (D) T3/T4N0 ( $\mathrm{P}=0.04)$. NS, not significant.

curative resection, PRL-3 overexpression in primary tumor was able to predict the poor outcome and was also an independent prognostic factor (Fig. 2B and Table IV). While, consistent with previous findings, PRL-3 overexpression did not have prognostic implication for 83 histologically nodepositive patients (23) (Fig. 2A). The explanation for this paradoxical phenomenon might be that PRL-3 overexpression may be associated with lymph node micrometastasis in histologically node-negative patients because of the strong correlation with lymph node metastasis. Advances in immunohistochemical and molecular biologic techniques have made it possible to identify microscopic residual disease, and UICC defines this residual disease as either isolated tumor cells or micrometastasis (33). Isolated tumor cells or micrometastasis in the lymph nodes could be identified in $17-41 \%$ of patients with histologically nodenegative gastric cancer $(34,35)$.

Interestingly, Yokoyama et al examined the biological and clinical significance of isolated tumor cells and micrometastasis in the lymph nodes, using animal model developed by inoculation of GCIY gastric cancer cells tagged with green fluorescent protein gene into nude mouse, and revealed that isolated tumor cells in the lymph nodes regressed spontaneously after resection of the primary tumor through immunologic activity conferred by NK cells, but micrometastasis proliferated (36). However, the micrometastasis could be partially eliminated by postoperative chemotherapy with $\mathrm{S}-1$, an oral fluoropyrimidine, at early stage, indicating the importance of adjuvant chemotherapy for lymph node micrometastasis. Therefore, we speculate that PRL-3 overexpression may represent the presence of lymph node micrometastasis in the histologically node-negative patients, inversely affecting the outcome. In contrast, in the histologically node-positive patients, PRL-3 overexpression may no longer have prognostic implications because of the presence of histological lymph node metastasis itself.

Collectively, these findings suggest that PRL-3 may be a key molecule related to lymph node metastasis, including micrometastasis, and be useful as an independent prognostic biomarker in histologically node-negative patients. In addition, PRL-3 overexpression was frequently detected not only in lymph node metastasis, but also in peritoneal metastasis in gastric cancer $(10,22-24)$. Moreover, SGC7901 gastric cancer cells with PRL-3 knockdown induced by artificial miRNA based on the murine miR-155 sequence significantly depressed the invasion and migration properties in vitro, and suppressed the growth of peritoneal metastases in nude mice (37). PRL-3 overexpression may be also associated with peritoneal metastasis.

Systemic therapy is fundamental to control occult metastasis in gastric cancer (38), and the Adjuvant Chemotherapy Trial of TS-1 for Gastric Cancer (ACTS-GC) has shown that 
S-1 monotherapy after curative surgery is effective to improve the overall survival and relapse-free survival of patients with stage II and III disease according to JCGC (39). Although patients with stage I disease after curative resection have good prognosis, especially early gastric cancer regardless of lymph node metastasis status, some patients still die due to disease recurrence (40). Stage I disease includes advanced gastric cancer without lymph node metastasis (T2N0), among which gastric cancers with invasion of the subserosa are reported to have unfavorable prognosis with 5-year DSS of $77.2 \%$ (41). However, such patients have not received adjuvant chemotherapy, and it is, therefore, indispensable to select patients with stage I disease who will benefit from adjuvant chemotherapy in clinical practice.

In the present study, it is particularly noteworthy that in advanced gastric cancer with stage I disease, PRL-3 overexpression showed a characteristic of stage II disease from a prognostic point of view (Fig. 2C), indicating a potential utility as a clinical applicable biomarker in considering adjuvant chemotherapy for advanced gastric cancer with stage I disease. These patients might be up-graded from stage I disease to stage II disease by the presence of lymph node micrometastasis. Further research will be necessary to validate the potential clinical effect of these results.

PRL-3 has deserved attention as a molecule associated with metastasis in various tumor types, and has been intensively examined in order to elucidate the metastatic mechanism. In DLD-1 colorectal cancer cells, enforced PRL-3 expression up-regulates the phosphoinositide 3-kinase (PI3K) pathway through down-regulation of phosphatase and tensin homologue deleted chromosome 10 (PTEN), thereby leading to epithelialmesenchymal transition (EMT) (42).

EMT is an important process during tumor metastasis by which epithelial cells acquire mesenchymal fibroblast-like properties and show reduced intercellular adhesion and increased motility (43). In SWS480 colorectal cancer cells, the enforced PRL-3 expression activates Rho family GTPases to promote invasion and motility (44). In HEK 293 embryonic kidney cells, the enforced PRL-3 expression induces Src activation through down-regulation of C-terminal Src kinase (Csk), and initiates phosphorylation of the extracellular signalregulated protein kinase 1/2 (ERK1/2), signal transducer and activator of transcription 3 (STAT3), and p130 Cas, thereby promoting cell growth and motility (45). Moreover, PRL-3 initiates tumor angiogenesis by recruiting endothelial cells (18), and may produce proteases to digest the basal lamina of the nearby blood vessels for intravasation (16). These findings indicate that PRL-3 might comprehensively play crucial roles in the multiple processes of metastasis and be a potential therapeutic target to prevent these processes (46-48).

In summary, we have demonstrated for the first time that PRL-3 expression in primary tumor could predict the outcome of patients with histologically node-negative gastric cancer. We have also shown that PRL-3 expression could have a potential utility as a clinically applicable biomarker that may facilitate the development of adjuvant chemotherapy for advanced gastric cancer with stage I disease. We believe that further studies assessing the value and potential applications of PRL-3 as a biomarker in gastric cancer are warranted.

\section{Acknowledgements}

This work was supported in part by the Grant-in-Aid for Cancer Research from the Ministry of Health, Labour and Welfare of Japan and by the Japanese Foundation for Multidisciplinary Treatment of Cancer.

\section{References}

1. Crew KD and Neugut AI: Epidemiology of gastric cancer. World J Gastroenterol 12: 354-362, 2006.

2. Parkin DM, Bray F, Ferlay J and Pisani P: Global cancer statistics, 2002. CA Cancer J Clin 55: 74-108, 2005.

3. Pantel $\mathrm{K}$ and Brakenhoff RH: Dissecting the metastatic cascade. Nat Rev Cancer 4: 448-456, 2004.

4. Bessette DC, Qiu D and Pallen CJ: PRL PTPs: mediators and markers of cancer progression. Cancer Metastasis Rev 26: 231252,2008

5. Zeng Q, Si X, Horstmann H, Xu Y, Hong W and Pallen CJ: Prenylation-dependent association of protein-tyrosine phosphatases PRL-1, -2 , and -3 with the plasma membrane and the early endosome. J Biol Chem 275: 21444-21452, 2000.

6. Zeng Q, Hong W and Tan YH: Mouse PRL-2 and PRL-3, two potentially prenylated protein tyrosine phosphatases homologous to PRL-1. Biochem Biophys Res Commun 244: 421-427, 1998.

7. Matter WF, Estridge T, Zhang CJ, et al: Role of PRL-3, a human muscle-specific tyrosine phosphatase, in angiotensin-II signaling. Biochem Biophys Res Commun 283: 1061-1068, 2001.

8. Dumaual CM, Sandusky GE, Crowell PL and Randall SK: Cellular localization of PRL-1 and PRL-2 gene expression in normal adult human tissues. J Histochem Cytochem 54: 1401-1412, 2006.

9. Saha S, Bardelli A, Buckhaults P, et al: A phosphatase associated with metastasis of colorectal cancer. Science 294: 1343-1346, 2001.

10. Miskad UA, Semba S, Kato H and Yokozaki H: Expression of PRL-3 phosphatase in human gastric carcinomas: close correlation with invasion and metastasis. Pathobiology 71: 176-184, 2004.

11. Polato F, Codegoni A, Fruscio R, et al: PRL-3 phosphatase is implicated in ovarian cancer growth. Clin Cancer Res 11: 6835-6839, 2005

12. Radke I, Gotte M, Kersting C, Mattsson B, Kiesel L and Wulfing P: Expression and prognostic impact of the protein tyrosine phosphatases PRL-1, PRL-2, and PRL-3 in breast cancer. Br J Cancer 95: 347-354, 2006.

13. Fagerli UM, Holt RU, Holien T, et al: Overexpression and involvement in migration by the metastasis-associated phosphatase PRL-3 in human myeloma cells. Blood 12: 806-815, 2007.

14. Kong L, Li Q, Wang L, Liu Z and Sun T: The value and correlation between PRL-3 expression and matrix metalloproteinase activity and expression in human gliomas. Neuropathology 27: 516-521, 2007.

15. Zeng Q, Dong JM, Guo K, et al: PRL-3 and PRL-1 promote cell migration, invasion, and metastasis. Cancer Res 63: 2716-2722, 2003.

16. Guo K, Li J, Tang JP, Koh V, Gan BQ and Zeng Q: Catalytic domain of PRL-3 plays an essential role in tumor metastasis: formation of PRL-3 tumors inside the blood vessels. Cancer Biol Ther 3: 945-951, 2004.

17. Wu X, Zeng H, Zhang X, et al: Phosphatase of regenerating liver-3 promotes motility and metastasis of mouse melanoma cells. Am J Pathol 164: 2039-2054, 2004.

18. Guo K, Li J, Wang H, et al: PRL-3 initiates tumor angiogenesis by recruiting endothelial cells in vitro and in vivo. Cancer Res 66: 9625-9635, 2006.

19. Rouleau C, Roy A, St Martin T, et al: Protein tyrosine phosphatase PRL-3 in malignant cells and endothelial cells: expression and function. Mol Cancer Ther 5: 219-229, 2006.

20. Kato H, Semba S, Miskad UA, Seo Y, Kasuga M and Yokozaki H: High expression of PRL-3 promotes cancer cell motility and liver metastasis in human colorectal cancer: a predictive molecular marker of metachronous liver and lung metastases. Clin Cancer Res 10: 7318-7328, 2004.

21. Qian F, Li YP, Sheng X, Zhang ZC, et al: PRL-3 siRNA inhibits the metastasis of B16-BL6 mouse melanoma cells in vitro and in vivo. Mol Med 13: 151-159, 2007. 
22. Miskad UA, Semba S, Kato H, et al: High PRL-3 expression in human gastric cancer is a marker of metastasis and grades of malignancies: an in situ hybridization study. Virchows Arch 450: 303-310, 2007.

23. Li ZR, Wang Z, Zhu BH, et al: Association of tyrosine PRL-3 phosphatase protein expression with peritoneal metastasis of gastric carcinoma and prognosis. Surg Today 37: 646-651, 2007.

24. Wang Z, He YL, Cai SR, et al: Expression and prognostic impact of PRL-3 in lymph node metastasis of gastric cancer: its molecular mechanism was investigated using artificial microRNA interference. Int J Cancer 123: 1439-1447, 2008.

25. Nakajima T: Gastric cancer treatment guidelines in Japan. Gastric Cancer 5: 1-5, 2002.

26. Japanese Gastric Cancer Association: Japanese classification of gastric carcinoma - 2nd English edition - response assessment of chemotherapy and radiotherapy for gastric carcinoma: clinical criteria. Gastric Cancer 4: 1-8, 2001.

27. Sobin $\mathrm{LH}$ and Wittekind $\mathrm{CH}$ : International Union Against Cancer (UICC): TNM Classification of Malignant Tumor. 5th edition, John Wiley and Sons, New York, 1997.

28. Hatate K, Yamashita K, Hirai K, et al: Liver metastasis of colorectal cancer by protein-tyrosine phosphatase type 4A, 3 (PRL-3) is mediated through lymph node metastasis and elevated serum tumor markers such as CEA and CA19-9. Oncol Rep 20: 737-743, 2008.

29. Peto R, Pike MC and Armitage P: Design and analysis of randomized clinical trials requiring prolonged observation of each patient. II. Analysis and examples. Br J Cancer 35: 1-39, 1977.

30. Harrell FE Jr, Lee KL and Pollock BG: Regression models in clinical studies: determining relationships between predictors and response. J Natl Cancer Inst 80: 1198-1202, 1988.

31. Siewert JR, Bottcher K, Stein HJ and Roder JD: Relevant prognostic factors in gastric cancer: ten-year results of the German Gastric Cancer Study. Ann Surg 228: 449-461, 1998.

32. Hartgrink HH, van de Velde CJ, Putter H, et al: Extended lymph node dissection for gastric cancer: who may benefit? Final results of the randomized Dutch gastric cancer group trial. J Clin Oncol 22: 2069-2077, 2004.

33. Sobin LH: TNM, sixth edition: new developments in general concepts and rules. Semin Surg Oncol 21: 19-22, 2003.

34. Okada Y, Fujiwara Y, Yamamoto H, et al: Genetic detection of lymph node micrometastases in patients with gastric carcinoma by multiple-marker reverse transcriptase-polymerase chain reaction assay. Cancer 92: 2056-2064, 2001
35. Nakajo A, Natsugoe S, Ishigami S, et al: Detection and prediction of micrometastasis in the lymph nodes of patients with pN0 gastric cancer. Ann Surg Oncol 8: 158-162, 2001.

36. Yokoyama H, Nakanishi H, Kodera Y, et al: Biological significance of isolated tumor cells and micrometastasis in lymph nodes evaluated using a green fluorescent protein-tagged human gastric cancer cell line. Clin Cancer Res 12: 361-368, 2006.

37. Li Z, Zhan W, Wang Z, Zhu B, He Y, Peng J, Cai S and Ma J: Inhibition of PRL-3 gene expression in gastric cancer cell line SGC7901 via microRNA suppressed reduces peritoneal metastasis. Biochem Biophys Res Commun 348: 229-237, 2006.

38. Sasako M: Surgery and adjuvant chemotherapy. Int J Clin Oncol 13: 193-135, 2008.

39. Sakuramoto S, Sasako M, Yamaguchi T, et al: Adjuvant chemotherapy for gastric cancer with S-1, an oral fluoropyrimidine. N Engl J Med 357: 1810-1820, 2007.

40. Maruyama K, Kaminishi M, Hayashi K, et al: Gastric cancer treated in 1991 in Japan: data analysis of nationwide registry. Gastric Cancer 9: 51-66, 2006.

41. Park do J, Kong SH, Lee HJ, et al: Subclassification of pT2 gastric adenocarcinoma according to depth of invasion (pT2a vs. pT2b) and lymph node status (pN). Surgery 141: 757-763, 2007.

42. Wang H, Quah SY, Dong JM, Manser E, Tang JP and Zeng Q: PRL-3 down-regulates PTEN expression and signals through PI3K to promote epithelial-mesenchymal transition. Cancer Res 67: 2922-2926, 2007.

43. Peinado H, Olmeda D and Cano A: Snail, Zeb and bHLH factors in tumour progression: an alliance against the epithelial phenotype? Nat Rev Cancer 7: 415-428, 2007.

44. Fiordalisi JJ, Keller PJ and Cox AD: PRL tyrosine phosphatases regulate rho family GTPases to promote invasion and motility. Cancer Res 66: 3153-3161, 2006.

45. Liang F, Liang J, Wang WQ, Sun JP, Udho E and Zhang ZY: PRL3 promotes cell invasion and proliferation by downregulation of Csk leading to Src activation. J Biol Chem 282: 5413-5419, 2007.

46. Dursina B, Reents R, Delon C, et al: Identification and specificity profiling of protein prenyltransferase inhibitors using new fluorescent phosphoisoprenoids. J Am Chem Soc 128: 2822-2835, 2006.

47. Choi SK, Oh HM, Lee SK, et al: Biflavonoids inhibited phosphatase of regenerating liver-3 (PRL-3). Nat Prod Res 20: 341-346, 2006.

48. Ahn JH, Kim SJ, Park WS, et al: Synthesis and biological evaluation of rhodanine derivatives as PRL-3 inhibitors. Bioorg Med Chem Lett 16: 2996-2999, 2006. 